\title{
Stage IIB Lung Cancer AJCC v8
}

National Cancer Institute

\section{Source}

National Cancer Institute. Stage IIB Lung Cancer A/CC V8. NCI Thesaurus. Code C136477.

Stage IIB includes: (T1a, N1, M0); (T1b, N1, M0); (T1c, N1, M0); (T2a, N1, M0); (T2b, N1, M0); (T3, NO, M0). T1a: Tumor measuring $1 \mathrm{~cm}$ or less in greatest dimension. A superficial, spreading tumor of any size whose invasive component is limited to the bronchial wall and may extend proximal to the main bronchus also is classified as T1a, but these tumors are uncommon. T1 b: T umor measuring more than $1 \mathrm{~cm}$ but $2 \mathrm{~cm}$ or less in greatest dimension. T1c: Tumor measuring more than $2 \mathrm{~cm}$ but $3 \mathrm{~cm}$ or less in greatest dimension. T2a: Tumor measuring more than $3 \mathrm{~cm}$ but $4 \mathrm{~cm}$ or less in greatest dimension. T2b: T umor measuring more than $4 \mathrm{~cm}$ but $5 \mathrm{~cm}$ or less in greatest dimension. T3: T umor measuring more than $5 \mathrm{~cm}$ but $7 \mathrm{~cm}$ or less in greatest dimension or directly invading any of the following: parietal pleura (PL3), chest wall (including superior sulcus tumors), phrenic nerve, parietal pericardium; or separate tumor nodule(s) in the same lobe as the primary. N0: No regional lymph node metastases. N1: Metastasis in ipsilateral peribronchial and/or ipsilateral hilar lymph nodes and intrapulmonary nodes, including involvement by direct extension. M0: No distant metastasis. (AJCC 8th ed.) 